\title{
La función de la biblioteca penitenciaria en la reinserción social de los internos del Complejo Penitenciario Puente Grande, Jalisco, México
}

\section{Julián Ochoa-García}

Universidad Panamericana. Escuela de Pedagogía. Álvaro del Portillo 49, Zapopan, Jalisco, 45010, México I jochoa@up.edu.mx / https://orcid.org/oooo-0oo1-816o-4313

\section{Humberto Martínez-Camacho}

Universidad Panamericana. Biblioteca. Álvaro del Portillo 49, Zapopan, Jalisco, 45010, México I

hmartinezc@up.edu.mx / https://orcid.org/oooo-0001-5430-0648

\section{Resumen}

En el presente trabajo se analiza la influencia que tienen las bibliotecas dentro de los centros penitenciarios, en relación con la readaptación social de sus internos. El artículo aborda la perspectiva de los propios responsables de las bibliotecas o espacios de lectura dentro de los centros carcelarios que componen el Complejo Penitenciario de "Puente Grande", en el Estado de Jalisco, México, por medio de un cuestionario que fue aplicado a los encargados de las bibliotecas de los cuatro centros que componen dicho complejo, en el cual se abordan, desde la perspectiva cualitativa, el concepto de biblioteca y su influencia en la readaptación social, el análisis de los servicios prestados y por último la atención y formación de usuarios. Entre los principales resultados se rescatan: 1) la importancia de la actividad lectora y formativa como instrumento para la reinserción social, 2) la falta de formación profesional para que los encargados, así como 3) la deficiencia en cuanto a los recursos físicos y tecnológicos.

The role of the penitentiary library in the social rehabilitation of inmates of the "Puente Grande" Penitentiary Complex, Jalisco, Mexico
Palabras clave

Bibliotecas penitenciarias Reinserción social Centros penitenciarios México

\begin{abstract}
This work analyzes the influence that libraries have within penitentiary centers in relation to the social rehabilitation of their inmates from the perspective of those responsible for libraries or reading spaces within the prisons that compose the "Puente Grande" Penitentiary Complex, in the State of Jalisco, Mexico, carrying out a questionnaire that was applied to those in charge of the libraries in the four centers that are part of this complex, in which the library concept and its influence on social readaptation from a qualitative perspective, the analysis of the services provided and, finally, the care and training of users. Among the main results, the following are highlighted: 1) the importance of reading and training activities as an instrument
\end{abstract}

Keywords

Penitentiary libraries Social reintegration Penitentiary centers México 
for social reinsertion, 2) the lack of professional training for those in charge, of the libraries 3) the deficiency in terms of physical and technological resources.

Artículo recibido: 15-05-2020. Aceptado: 23-10-2020

\section{Introducción}

Desde los inicios de la existencia del hombre, la biblioteca ha jugado un papel importante en la conservación y generación del conocimiento humano, de tal manera que en ellas se atesore la memoria del saber humano a través del paso de los años. La figura del bibliotecario en este contexto es vital, ya que es quien preserva, administra, organiza y ofrece el contenido resguardado bajo su cargo para todo aquel miembro de la sociedad que requiera acceder al mismo. Bajo este tenor, dentro de las responsabilidades del bibliotecario "el principal es el compromiso informativo y social de los lectores; la utilización de recursos gestionados con eficiencia y el acercamiento a políticas públicas que sean activas para una sociedad más justa" (Marcos Recio, 2018: 158).

Es muy común que se conciba la función de los bibliotecarios como meros prestadores de servicios y como organizadores de la información ya existente, sin que aporten nada más al cúmulo del mundo del conocimiento y a su aplicación al mundo real, cuando la tarea de dichos profesionales tiene una función mucho más importante que el de sólo atesorar conocimientos y escritos valiosos. Tocando el tema sobre los prejuicios acerca del estereotipo de la profesión bibliotecaria, Clemente Castro retoma el trabajo de Torres Reyes (2011) quién afirma que "la profesión bibliotecaria no goza de buen prestigio ni es reconocida por la sociedad en general, ya que se sigue creyendo que la profesión se limita al préstamo de libros y que no es necesario estudiar a nivel universitario" (Clemente Castro, 2019: 23).

El objetivo de este trabajo se centra en analizar, con un panorama claro, la influencia que tienen las bibliotecas y los bibliotecarios dentro de los centros penitenciarios, en relación con la readaptación social de los internos. La idea central de la investigación es esclarecer de qué manera, los encargados perciben su rol como agentes de cambio en los centros penitenciarios, para buscar la readaptación social de los internos, por medio de los servicios que brinda la propia biblioteca.

De manera tradicional, se considera a la biblioteca como un espacio físico que cuenta con un acervo de material, principalmente impreso, en el cual se almacenan una serie de recursos que pueden ser consultados por los usuarios de las mismas. Pero la función de las bibliotecas va mucho más allá que ser solamente un almacén de libros y revistas. La figura del bibliotecario no se limita únicamente a mantener en orden el material existente, sino que tiene una función mucho más rica, al ser un verdadero intermediario entre las necesidades informativas de los usuarios y el conocimiento existente en las colecciones con que se cuenta en la biblioteca. Así pues, el profesional de las bibliotecas "está enfrentándose a un nuevo desafío para adaptarse al entorno digital y la supervivencia de las bibliotecas recae en el reto de la combinación de la faceta tradicional con la vertiente digital" (Clemente Castro, 2019: 33).

Concretamente, en el caso de los centros penitenciarios, es de vital importancia tener claro a qué dedican su tiempo las personas que se encuentran como responsables de las bibliotecas dentro de dichos centros. Definitivamente, la función de los encargados en los centros penitenciarios es el ser un verdadero apoyo para la readaptación integral de los internos, de manera que les ayuden a meditar sobre las faltas cometidas 
y que los han llevado a una situación de privación de la libertad. La tarea de los bibliotecarios dentro de una prisión "consiste en satisfacer las necesidades educativas, recreativas e informativas de los reclusos", dicho de otra manera, deben de "servir de apoyo a la educación reglada, a los programas de rehabilitación, al aprendizaje independiente y al estudio en general" (Ruibal, 2018: 34).

El objetivo del tiempo que los internos infractores pasan dentro de los centros penitenciarios debe consistir en devolver a la sociedad a unos sujetos readaptados, que puedan aportar al bien común de la sociedad a la que regresan una vez que han cumplido con su tiempo de condena. El castigo se considera desde la perspectiva jurídica "como aquel acto por el cual se persuade al infractor de una norma a no cometer acciones no deseadas por la sociedad" (Cisneros, 2016: 193), pero este autor reflexiona sobre si dicho confinamiento penitenciario, en sí mismo, puede resolver el problema de la infracción a la ley cometida por el sujeto que se encuentra privado de su libertad.

En este trabajo se buscó llevar a cabo una reflexión sobre la influencia que pueden tener las bibliotecas dentro de la función de los centros de readaptación social en la reinserción de los sujetos infractores, para así conocer su apoyo a dicha finalidad, por medio de los servicios que ofertan a la comunidad penitenciaria. De aquí surge la pregunta central de este trabajo, ¿de qué manera influyen los encargados de las bibliotecas penitenciarias en la tarea de la reinserción de los sujetos infractores en el Complejo Penitenciario Puente Grande, en el Estado de Jalisco?

\section{El sistema penitenciario}

Desde la aparición del hombre en la faz de la tierra, este ha buscado vivir en un entorno seguro y adecuado que le permita desarrollarse de manera integral en todos los aspectos de su vida. Parte esencial del hombre está regida por su naturaleza social, tal como lo afirma el propio Aristóteles, el hombre es un ser político por naturaleza, es decir, es parte de un grupo social (Aristóteles, 2000: 4). Por tanto, al vivir en comunidad es importante regular de alguna manera el comportamiento individual de cada uno de sus miembros de esa sociedad en aras de la consecución del bien común; en este sentido es que Rousseau propone que por medio de un pacto social se da origen a un Estado de Derecho que proteja y garantice la libertad de cada uno de los individuos que conforman al grupo social (Rousseau, 2014: 66).

Es importante que en la búsqueda de ese bien común que otorga seguridad social, no se vea perdida la libertad individual, sino que se regule correctamente la convivencia de los seres humanos unidos en un grupo social; justamente es la razón de ser de un correcto Estado de Derecho. En el contexto de un estado regido por el derecho, se deben considerar los elementos para contener los delitos que causen daño o vayan en contra de una sana convivencia entre los miembros de la comunidad; y es aquí donde se debe considerar la función del Derecho Penal, y más concretamente de una adecuada política criminal. Dicha política criminal, de acuerdo a Muñoz Blanco, se entiende como "la disciplina que se ocupa de cómo configurar el derecho penal de la forma más eficaz posible para que pueda cumplir con su tarea de protección de la sociedad" (Muñoz Blanco, 2004: 78).

\section{La readaptación social}

De acuerdo a Meza, la libertad del ser humano es una nota fundamental de su desarrollo en la búsqueda de uno de sus fines más importantes, es decir, la felicidad. Pero hace la aclaración de que esa libertad no debe ser confundida con el libertinaje y, por 
tanto, el actuar del ser humano requiere del cumplimiento de las reglas de conducta sociales, de las cuales el Estado es el garante. En la República Mexicana, la institución responsable del "internamiento de las personas que por motivo de su conducta antisocial tienen que compurgar una pena de carácter privativo de la libertad" son los Centros de Readaptación Social (CERESO) (Meza, 1978: 10). Así pues, el objeto que persiguen dichos centros "es la readaptación social del individuo para que cuando recobre su libertad vuelva a ser un ente social útil a la comunidad" (Meza, 1978: 10).

Por su parte, Madrazo asegura que "uno de los principales obstáculos para el desarrollo de cualquier nación lo constituye el bajo índice educativo de sus habitantes" (Madrazo, 1985: 7), y relaciona directamente en su trabajo esa deficiencia en la educación de los individuos como causa de los delitos cometidos por los mismos. Es por eso que, en su obra rescata la educación de los sujetos infractores como parte fundamental de su readaptación social. Aquí es donde se conecta directamente la importancia de contar con herramientas adecuadas para lograr una adecuada readaptación dentro de los centros penitenciarios, y la biblioteca es uno de los elementos indispensables para fomentar, por medio de la educación y la cultura, esa corrección integral de los internos.

En México se garantiza, dentro del ordenamiento legal, la obligación implícita de asegurar la readaptación social de los sujetos infractores que se encuentran internados dentro del propio sistema carcelario. Así pues, el artículo 14 de la Ley Nacional de Ejecución Penal establece que la autoridad penitenciaria:

La autoridad penitenciaria organizará la administración y operación del sistema penitenciario sobre la base del respeto a los derechos humanos, el trabajo, la capacitación para el mismo, la educación, la salud y el deporte, como medios para procurar la reinserción de la persona sentenciada a la sociedad y procurar que no vuelva a delinquir. (México. Ley Nacional de Ejecución Penal, 2016: 10)

También, en su artículo 72 de las bases de la organización señala que:

Son bases de la organización del sistema penitenciario para lograr la reinserción social: el respeto a los derechos humanos, el trabajo, la capacitación para el mismo, la educación, la salud y el deporte. Estas bases serán elementos esenciales del Plan de Actividades diseñado para las personas privadas de su libertad en los Centros Penitenciarios. (México. Ley Nacional de Ejecución Penal, 2016)

Asimismo, la misma ley en su artículo 83, a su vez hace alusión al artículo $3^{\circ}$ de la Constitución Política sobre el tema de los derechos humanos, en donde se plantea el derecho a la educación (México. Constitución Política de los Estados Unidos Mexicanos, 2020).

Una vez establecido el funcionamiento del sistema penitenciario en relación con la readaptación social, en los siguientes párrafos se analiza el concepto de la biblioteca como un agente de cambio social en los sujetos recluidos en los centros penitenciarios.

\section{Las bibliotecas como agentes de cambio social}

La biblioteca es el organismo más democrático, en el sentido de que es una institución que permite el uso del conocimiento generado por el ser humano, desde sus primeros orígenes, hasta los descubrimientos más recientes y novedosos en el campo del saber. 
Para efectos del presente escrito, se abordará la figura de la biblioteca pública en razón de que los servicios bibliotecarios existentes dentro de los centros penitenciarios pertenecen al ámbito social y público, al tratarse de instituciones gubernamentales que regulan el cumplimiento de las penas de los sujetos infractores como parte de su función social.

\section{Biblioteca pública}

De acuerdo con las Directrices de la IFLA/UNESCO para el desarrollo del servicio de bibliotecas públicas, ambos organismos rectores en el quehacer de las bibliotecas y los bibliotecarios, se asume a la biblioteca pública como una:

Organización establecida, respaldada y financiada por la comunidad, ya sea por conducto de una autoridad u órgano local, regional o nacional, o mediante cualquier forma de organización colectiva. Brinda acceso al conocimiento, a la información y al trabajo intelectual a través de una serie de recursos y servicios y está a disposición de todos los miembros de la comunidad en igualdad de condiciones sin distinción de raza, nacionalidad, edad, sexo, religión, idioma, discapacidad, condición económica y laboral y nivel de escolaridad (Gill, 2002: 11).

Siguiendo con el mismo texto de las Directrices de la IFLA/UNESCO, la biblioteca pública tiene como objetivo facilitar recursos informativos y prestar servicios mediante diversos medios para atender necesidades educativas, informativas y de desarrollo personal; ofrecen a la persona acceso al conocimiento y contribuyen al mantenimiento de una sociedad democrática, y también brindan una identidad cultural y son espacios públicos de encuentro (Gill, 2002).

En lo relativo a los beneficiarios de los servicios otorgados por una biblioteca públi$\mathrm{ca}$, se incluyen de manera precisa a los reclusos de los centros penitenciarios en el Manifiesto de la UNESCO, al declarar lo siguiente:

La biblioteca pública presta sus servicios sobre la base de igualdad de acceso de todas las personas, independientemente de su edad, raza, sexo, religión, nacionalidad, idioma o condición social. Debe contar además con servicios específicos para quienes por una u otra razón no puedan valerse de los servicios y materiales ordinarios, por ejemplo, minorías lingüísticas, deficientes físicos y mentales, enfermos o reclusos (UNESCO; IFLA, 1994: 74).

Una vez aclarado el concepto de la biblioteca pública y su relación directa con las bibliotecas penitenciarias, se da ahora paso al análisis del concepto de estas últimas, así como sus características y sus funciones concretas dentro de los centros de readaptación social.

\section{Bibliotecas penitenciarias}

Como se puede inferir de los párrafos anteriores, la importancia de la biblioteca en el desarrollo integral del ser humano, juega un papel significativo, ya que no solamente apoya el proceso educativo de sus usuarios, sino que aporta elementos valiosos al desarrollo personal, en todas las áreas que integran al ser humano.

En el caso de los sujetos que están purgando una condena privativa de la libertad, la importancia de la biblioteca cobra un mayor relieve en su diario acontecer, ya que el hecho de encontrarse recluidos en un centro penitenciario, limita de manera muy considerable las actividades a las que puedan dedicar su tiempo; aunque, si bien es cierto que existen una serie de opciones en las prisiones, tales como talleres para el 
desarrollo de algún oficio, actividades físicas entre otras, el hecho de poder contar con una biblioteca, aumenta los horizontes intelectuales de los internos, acompañando su aislamiento con buenas lecturas y actividades culturales que los mantengan, de alguna manera, conectados con el mundo exterior. Tal como lo menciona Pérez Pulido:

Los servicios bibliotecarios de prisiones surgen como apoyo a las actividades educativas, pero también en el convencimiento de que la lectura en prisión contribuye al desarrollo de la personalidad del recluso y ayuda a aliviar la pena. Así ha sucedido desde principios del siglo XX, cuando, partiendo del carácter instructivo y edificante de la lectura, se ha considerado a ésta como un instrumento común de rehabilitación (Pérez Pulido, 2007: 73).

Por otra parte, señala el mismo autor que las normas internacionales que regulan los servicios bibliotecarios, tanto a nivel nacional como internacional, consideran los aspectos esenciales para el apoyo del recluso. Los principios fundamentales para las bibliotecas de prisiones que destaca, son los siguientes:

»El derecho de los reclusos a acceder a la información dentro y fuera de su entorno.

» La consideración de los servicios bibliotecarios como parte del programa sociocultural del centro penitenciario.

»El apoyo a las actividades educativas del centro, al desarrollo personal y cultural de los reclusos, a su formación para el empleo y a la ocupación de sus momentos de ocio y distracción.

» La creación de un modelo organizativo similar al de una biblioteca pública.

» La necesidad de evaluar periódicamente el servicio para comprobar si cumple con los objetivos propuestos (Pérez Pulido, 2007: 78).

En el año 2001 la sección de bibliotecas para Personas en Condiciones Desventajosas (LSDP por sus siglas en el idioma inglés) de la International Federation of Library Associations (IFLA), ofrece una orientación para el desarrollo de servicios especializados para la comunidad que no tienen posibilidad de acceder a servicios bibliotecarios convencionales como personas en hospitales y prisiones (Lehmann y Locke, 2007: 3). Estos autores argumentan que a medida que las sociedades modernas han ido adoptando formas más humanas y progresistas de ejercer la justicia penal y la encarcelación, de acuerdo con la Declaración Universal de Derechos Humanos de la ONU, su perspectiva ha cambiado del simple castigo a la educación, rehabilitación y el uso productivo de su tiempo (Lehmann y Locke, 2007:4).

Sugieren también que la biblioteca de prisión brinda normalidad en un entorno donde todo es sumamente controlado; también mencionan que en la biblioteca las personas son libres de hacer sus propias elecciones y que esta ofrece una conexión con el mundo exterior y puede brindar información útil para aquellos que se preparan para luego de ser liberados. Tal como se puede leer claramente en el siguiente párrafo:

Una persona por estar encarcelada no renuncia a su derecho a aprender y a tener acceso a la información; por tanto, la biblioteca de prisión debe ofrecer materiales y servicios semejantes a los de las bibliotecas públicas en el mundo "libre". Sólo se deben imponer restricciones en el acceso a los materiales de lectura cuando el mismo represente un peligro inminente para la seguridad de la prisión (Lehman y Locke, 2005: 4).

En este documento, se consideran diez pautas en las cuales se contemplan los siguientes aspectos: Alcance, Administración, Acceso, Instalación y equipamiento, Tecnología 
de la información, Personal, Presupuesto, Materiales de biblioteca, Servicios y programas y, por último, la Comunicación y marketing.

Estas pautas se aplican para todos los centros donde existan personas encarceladas y deben servir de base para el desarrollo de procedimientos bibliotecarios locales, en los que todos los reclusos puedan tener acceso a sus servicios, sin importar su clasificación de seguridad o ubicación dentro de la prisión.

Dentro de dichas pautas, se considera al espacio físico de la biblioteca como importante ya que la efectividad y el éxito de los servicios bibliotecarios dependen en gran medida de un entorno físico acogedor y cómodo, el cual debe estar diseñado específicamente para el uso, la fluidez del trabajo y la funcionalidad.

Por otra parte, y siguiendo con estos lineamientos, se hace referencia a que las personas encargadas de las bibliotecas de prisiones deben ser bibliotecarios con preparación profesional y técnica, con habilidades humanas e interpersonales para ofrecer un servicio directo y asistencia al usuario. Los sueldos y los beneficios del personal deben ser semejantes a los de los empleados de las bibliotecas públicas; la biblioteca de prisión puede emplear también a reclusos o a voluntarios de la comunidad recibiendo un amplio programa de orientación y adiestramiento laboral.

En cuanto al presupuesto, las pautas señalan que la autoridad responsable de la biblioteca debe asegurar que esta sea financiada como una unidad independiente; el bibliotecario principal debe estar a cargo de la elaboración y administración del presupuesto y, además, redactar un informe anual de actividades y resultados de la misma.

Por último, se sostiene que los materiales para la biblioteca deben incluir documentos impresos y en otros formatos; la colección tendrá que ser "balanceada" y debe gestionarse y conservar de acuerdo a normas profesionales (Lehmann y Locke, 2007).

\section{El Complejo Penitenciario Puente Grande en el Estado de Jalisco (México)}

El presente trabajo está orientado a indagar y analizar la percepción de los encargados de las bibliotecas dentro del grupo penitenciario más importante en el Estado de Jalisco, Puente Grande, Jalisco, México, en relación con la importancia que guarda en el contexto del centro de readaptación social, así como el uso de los servicios con que se cuenta desde la perspectiva del bibliotecario de dichos centros.

De acuerdo al sitio Web oficial del Estado de Jalisco, la Fiscalía General es la dependencia responsable de la Seguridad Pública y la Procuración de Justicia, en términos de lo que establece el artículo 21 de la Constitución Política de los Estados Unidos Mexicanos (Gobierno del Estado de Jalisco, 2020). Dicha Fiscalía tiene a su cargo al Ministerio Público para conducir las funciones de la investigación de los delitos, de la seguridad pública, de mantener el orden y la paz pública. Por otro lado, conduce el mando de las policías, así como el ejercicio de la acción penal y la reparación del daño. La propia Fiscalía es responsable también del sistema de reinserción social, protección civil y atención a víctimas, que es lo que compete a este trabajo de investigación. (Gobierno del Estado de Jalisco, 2020)

El Sistema Penitenciario del Estado de Jalisco está constituido por los siguientes centros penitenciarios, distribuidos en todo su territorio (ver Tabla I). 
Tabla I. Centros Penitenciarios de la Coordinación General Estratégica de Seguridad y Secretaría de Seguridad del Estado de Jalisco (2019)

\begin{tabular}{|l|l|c|}
\hline Centro penitenciario & Nombre de la biblioteca & $\begin{array}{c}\text { Número de } \\
\text { bibliotecas }\end{array}$ \\
\hline Comisaría de Prisión Preventiva & $\begin{array}{l}\text { Biblioteca de la Comisaría de Prisión } \\
\text { Preventiva }\end{array}$ & 1 \\
\hline Comisaría de Sentenciados & $\begin{array}{l}\text { Biblioteca de la Comisaría de Reinserción } \\
\text { Social }\end{array}$ & 1 \\
\hline Comisaría de Reinserción Femenil & Juan Nepomuceno Almonte & 1 \\
\hline Reclusorio Metropolitano & Biblioteca Gandhi & 1 \\
\hline $\begin{array}{l}\text { Centro de Atención Integral Juvenil del Estado } \\
\text { de Jalisco }\end{array}$ & Juan Rulfo & - \\
\hline $\begin{array}{l}\text { Centro de Observación, Clasificación y } \\
\text { Diagnóstico del Estado de Jalisco }\end{array}$ & $\begin{array}{l}\text { No cuenta con biblioteca establecida } \\
\text { formalmente pero si tiene 5oo libros para la } \\
\text { consulta de personas privadas de su libertad }\end{array}$ & \\
\hline Reclusorio de Puerto Vallarta & $\begin{array}{l}\text { Biblioteca de la Sección Femenil “Sor Juana } \\
\text { Inés de la Cruz”. } \\
\text { Biblioteca de la Sección Varonil 1 “Benito }\end{array}$ \\
\hline $\begin{array}{l}\text { Juárez García”. } \\
\text { Gentro Integral de Justicia Regional de Ciudad } \\
\text { Guzmán }\end{array}$ & Refugio Barragán Toscano & 3 \\
\hline Centro Integral de Justicia Regional de Tequila & Octavio Paz & 1 \\
\hline Centro Integral de Justicia Regional de Chapala & $\begin{array}{l}\text { Biblioteca del Centro Integral de Justicia } \\
\text { Regional de Chapala }\end{array}$ & 1 \\
\hline $\begin{array}{l}\text { Centro Integral de Justicia Regional de } \\
\text { Tepatitlan }\end{array}$ & Francisco Alcalá Barba & 1 \\
\hline Centro Integral de Justicia Regional de Autlán & Amozpoan & 1 \\
\hline Centro Integral de Justicia Regional de Moreno & Mariano Azuela & 1 \\
\hline
\end{tabular}

Fuente: Información proporcionada por la Unidad de Transparencia.

El Estado de Jalisco tiene catorce centros penitenciarios pertenecientes a la Dirección General de Reinserción social, cada uno de ellos cuenta con un espacio dedicado para los servicios bibliotecarios, a excepción de uno de ellos que no posee una biblioteca como tal, pero sin embargo sí presenta una colección de 500 títulos a disposición de los internos (Centro de Observación, Clasificación y diagnóstico del Estado de Jalisco). Por otro lado, el Reclusorio de Puerto Vallarta, cuenta con tres bibliotecas formales para los internos: Biblioteca de la Sección Femenil "Sor Juana Inés de la Cruz", Biblioteca de la Sección Varonil "Benito Juárez García” y Biblioteca de la Sección Varonil “Emiliano Zapata” (ver tabla I).

Para efectos del presente trabajo de investigación, se eligió al Centro Penitenciario Puente Grande, al ser el más relevante en tamaño e importancia en el Estado de Jalisco. Se contactó, tanto vía telefónica como por correo electrónico, al Director General de Reinserción Social del Estado para solicitar su apoyo en localizar a los encargados de cada una de las bibliotecas pertenecientes al mencionado complejo de "Puente Grande", compuesto por los siguientes centros: 
1. Comisaría de Prisión Preventiva

2. Comisaría de Sentenciados

3. Comisaría de Reinserción Femenil

4. Centro de Atención integral Juvenil del Estado de Jalisco.

De los cuatro centros anteriores se obtuvo la respuesta al instrumento enviado y sus datos se tomaron en cuenta para hacer el análisis de la influencia de la figura de la biblioteca como apoyo para la reinserción social de los internos en dichos centros penitenciarios. Se decidió realizar el trabajo de campo en el complejo penal Puente Grande, debido a que este es el más cercano a la Zona Metropolitana de la Ciudad de Guadalajara, y al ser el que concentra un mayor número de reclusos pertenecientes a esta población, que es la capital del Estado.

\section{Metodología}

Para el análisis de este trabajo se diseñó un instrumento desde la perspectiva del análisis cualitativo para conocer la opinión de los encargados de las bibliotecas sobre la influencia de las mismas en la reinserción social de los presos. El instrumento adecuado para medir la percepción de los bibliotecarios de dichos centros, en relación al aprovechamiento de los internos de dicho servicio, es el cuestionario.

El instrumento utilizado en la presente investigación, parte del planteamiento de Domínguez Sanjurjo en su texto "Nuevas formas de organización y servicios en la biblioteca pública". Esta autora describe la encuesta de la siguiente manera: "son métodos de recogida de información que, junto con los otros métodos ya señalados, permiten a la biblioteca conocer las necesidades de los usuarios reales y/o potenciales y el grado de adecuación de los servicios que ofrece" (Domínguez Sanjurjo, 1996: 61). Para este trabajo, la herramienta utilizada para la recogida de datos en investigaciones como la que aquí se presenta, es el cuestionario. En relación a dicho instrumento, Hernández Sampieri, Fernández Collado y Baptista Lucio expresan que el "cuestionario es un conjunto de preguntas respecto de una o más variables a medir". (2014: 217). Por medio de su aplicación se pueden obtener elementos de gran valor, ya que en ellos se expresan las opiniones de los sujetos que los responden, así como sus competencias y conocimientos sobre un tema concreto sobre el cual se trata de indagar (Colás, Buendía y Hernández, 2009: 70).

Además se realizó un análisis de las respuestas de los encargados de las bibliotecas y así identificar su opinión en relación al impacto de esta institución en el contexto de la readaptación social de los sujetos infractores que se encuentran internos en esos centros penitenciarios, por medio de las respuestas a dichos cuestionarios.

\section{Diseño del instrumento}

El diseño del cuestionario se compone de dos partes; en la primera se recopila la información general de cada centro, así como los datos del encargado de la biblioteca y en la segunda parte se presentan 17 ítems o preguntas, tanto de opción múltiple como preguntas abiertas.

Los temas abordados en dicho instrumento abarcan desde opiniones personales de los encargados de las bibliotecas, servicios ofertados, personal que se desempeña en ellas y horarios. Concretamente se preguntó sobre la capacitación y formación que los encargados perciben como necesaria para desempeñar correctamente su puesto; por último, se indagó sobre los cursos que ellos mismos ofrecen a la comunidad penitenciaria. En este último punto se busca identificar, desde la perspectiva de los 
encargados de las bibliotecas, cuáles son los aspectos positivos de esta agencia en el proceso de readaptación social de los internos.

\section{Resultados}

En este apartado se presenta el análisis de los resultados arrojados por los cuestionarios (C) aplicados a los encargados de las bibliotecas de los cuatro centros penitenciarios que componen al complejo "Puente Grande", así como su confrontación con las fuentes académicas que fundamentaron el marco teórico de este documento académico. A continuación, se presentan los resultados derivados de dicho análisis.

Como ya se había mencionado antes, el cuestionario fue respondido por los encargados de las bibliotecas del complejo penitenciario Puente Grande en el Estado de Jalisco. En relación al género, existe una igualdad entre hombres y mujeres, al contar con el $50 \%$ de representatividad en el puesto. Con respecto a la edad, hay una gran diversidad en los bibliotecarios, ya que dos de ellos se encuentran en el rango de 30 a 40 años, mientras que uno de los restantes está incluido en el grupo de 50 a 60, y el último de ellos en el rango de los 60 a los 70 años. En relación con la formación profesional de los encargados de las bibliotecas, se puede constatar que ninguno de los cuatro sujetos había estudiado alguna carrera relacionada con la biblioteconomía o alguna carrera afín, de manera que sus áreas de formación son en otras áreas del conocimiento.

Derivado de las respuestas obtenidas por medio de los instrumentos aplicados a los responsables, se pueden identificar tres grandes apartados en relación a la importancia de la biblioteca en el contexto de los centros de readaptación social:

1. El concepto de biblioteca y su influencia en la readaptación social.

2. El análisis de los servicios prestados.

3. Atención y formación de usuarios.

Para lo referente al concepto de biblioteca y su influjo en la readaptación social, se cuestionó a los encargados sobre los siguientes aspectos:

Al cuestionarlos sobre la propia concepción de la figura de la biblioteca, se puede observar en las respuestas del $75 \%$ de ellos, la relevancia que tiene para ellos la actividad lectora en su formación. Así, en una de ellas se puede leer lo siguiente:

"Un santuario. Un lugar para recrear, alimentar nuestra mente, nuestra conciencia, un lugar que edifica, alimenta, expande la mente ya que nos regala conocimientos. Viajamos, conocemos a través de los libros que nos ofrece la biblioteca con todas sus colecciones". (C-2)

Es importante aquí recordar lo establecido en las Directrices de la IFLA/UNESCO, al afirmar que la biblioteca pública tiene como objetivo facilitar recursos informativos y prestar servicios mediante diversos medios para atender necesidades educativas, informativas y de desarrollo personal (Gill, 2002). Es reconfortante verificar que dentro del concepto de biblioteca que tienen los encargados en los centros penitenciarios, existe una coincidencia con las mencionadas directrices en cuanto al sentido principal en relación a los usuarios.

En cuanto a la importancia de la biblioteca en el proceso de reinserción social de los internos, las respuestas de los encargados muestran nuevamente la importancia que reconocen a la lectura como elemento importante de apoyo al proceso de reinserción social, ya que todas las respuestas se orientan a dicho aporte de la lectura como 
actividad principal de las bibliotecas penitenciarias. Entre los beneficios mencionados en dichas respuestas, resaltan la identificación con los autores de los libros leídos por los presos (C-4), el acceso a información valiosa por medio de la lectura para fomentar el aprendizaje (C-2), la reflexión personal de los lectores al momento de mantener un "diálogo" con los libros (C-4), la obtención de herramientas personales para afrontar los embates del día a día (C-3), entre otros beneficios que concede el ejercicio de la lectura en los internos.

Aquí es conveniente retomar una de las afirmaciones de Pérez Pulido, al afirmar que los servicios de las bibliotecas penitenciarias, además de apoyar la labor educativa de los usuarios, también fomentan por medio de la lectura el desarrollo de la personalidad del recluso y adicionalmente, le ayuda a aliviar la pena causada por la privación de su libertad (Pérez Pulido, 2007: 73).

En lo concerniente a la formación profesional, la totalidad de los encargados de las bibliotecas penitenciarias afirman no haber recibido una capacitación profesional para desempeñar su labor en los centros penitenciarios. Llama poderosamente la atención que tampoco hayan sido capacitados formalmente para atender al tipo de usuarios tan especiales que hacen uso de dichas bibliotecas, pues, en definitiva, los sujetos privados de la libertad en razón de haber cometido algún tipo de ilícito, son un tipo de usuarios que requieren de una atención especial por su propia situación y contexto en que se desarrollan.

En definitiva, se requiere de una labor especialmente enfocada a la educación por parte de los encargados de dichas bibliotecas, pues como se menciona al inicio de éste trabajo, una de las situaciones que constituyen un verdadero impedimento para la readaptación social de los internos en los centros penitenciarios es su bajo nivel educativo (Madrazo, 1985), lo que genera de alguna manera una falta de conciencia en su actuar, y puede llevar con mayor facilidad a la comisión de delitos por los mismos. Aquí es dónde cobra importancia el promover la mejora educativa de los presos, por medio de las actividades propuestas por los encargados de las bibliotecas.

Respecto al análisis de los servicios prestados en las bibliotecas objeto del presente análisis, se cuestionó a los encargados sobre la pertinencia de los recursos bibliotecarios que cuentan en sus instalaciones, a lo cual respondieron los cuatro encargados que sus bibliotecas cuentan con la infraestructura física básica; es decir, catálogo público impreso, colección de libros, salas de lectura, mesas y sillas de trabajo; únicamente dos de ellos afirma contar con una computadora como apoyo a sus labores, pero sin acceso a Internet (C-1 y C-4), y en ninguno de los casos se cuenta con equipo de cómputo para los usuarios. Como se puede observar, las bibliotecas analizadas, cuentan con los servicios básicos en cuanto a la infraestructura física, pero no así en los apoyos tecnológicos para los usuarios y los propios encargados. Se puede deducir que, de acuerdo a los servicios identificados, el enfoque de servicio de dichas bibliotecas está dirigido básicamente a la lectura en material impreso y a asegurar un mínimo de estancia de los usuarios por medio de mobiliario básico (mesas y sillas).

Si recordamos aquí las pautas para servicios bibliotecarios para reclusos, propuestas por la IFLA, en la pauta número 5 , se hace referencia a la importancia de contar con el apoyo de las tecnologías de la información; se resalta la conveniencia de que los usuarios cuenten con acceso a computadores con servicios multimedia y, siempre que la seguridad del centro penitenciario no se vea afectada, se recomienda ofrecer el servicio de acceso a internet (Lehmann y Locke, 2007).

Como se puede observar, en las bibliotecas analizadas, es mínimo el apoyo tecnológico con que cuentan los encargados de las mismas. Sin embargo, la parte toral de 
la finalidad de las bibliotecas penitenciarias sí se cumple, al ofrecer los servicios de acceso al material de lectura y al mobiliario mínimo para garantizar las condiciones adecuadas para dicha actividad lectora.

Por último, en cuanto a la atención y formación de usuarios, la totalidad de los encargados de las bibliotecas dicen ofrecer cursos y actividades de formación para los usuarios que los visitan. Entre la oferta de dichas bibliotecas, los encargados mencionan que se ofrecen las siguientes: torneos de ajedrez, proyección de películas (C-1), cursos de literatura (C-2) y círculos de lectura (C-4). Si acudimos nuevamente a las pautas para servicios bibliotecarios para reclusos de la IFLA, se observa la importancia de los cursos formativos en las funciones de una biblioteca penitenciaria, tal como se menciona en el punto número 9:

"La biblioteca debe organizar y apoyar diversas actividades y programas que promuevan la lectura, la alfabetización y la cultura. Tales programas ofrecen la oportunidad de emplear el tiempo de forma creativa y mejorar la calidad de vida, también fomentan las habilidades sociales y una mayor autoestima. Las autoridades de la prisión deben tener en cuenta que los reclusos que están ocupados en actividades útiles son menos propensos a provocar problemas y disturbios" (Lehmann y Locke, 2007: 16).

Una vez analizados los principales aspectos que confieren relevancia a las bibliotecas dentro de los centros penitenciarios, así como la importancia que juegan los propios encargados de las mismas en la readaptación social de los internos, se da paso a las siguientes conclusiones.

\section{Conclusiones}

Para culminar el presente trabajo de investigación, y después de analizar la relevancia de las bibliotecas dentro de los centros penitenciarios, se puede inferir que es sumamente significativo conocer la importancia, sobre todo de generar conciencia, de la necesidad de contar con servicios bibliotecarios dentro de los centros penitenciarios.

La función esencial de los centros penitenciarios es la búsqueda de la readaptación social de los internos. Esta readaptación requiere de diversos apoyos para lograr su fin; no solamente de la parte punitiva de la privación de la libertad de los sujetos que infringen la ley, sino también de una reeducación de los internos. La biblioteca es un apoyo esencial para lograr esa parte educativa que implica la readaptación social.

En el Estado de Jalisco, se cumple con la función primordial que se pretende lograr al contar con bibliotecas dentro de los centros penitenciarios, es decir, brindar el apoyo para la reeducación de los presos, por medio de su elemento toral, la promoción de la lectura como base mínima de la educación. Sin embargo, es necesario considerar las recomendaciones de organismos nacionales e internacionales que son autoridad en el campo de las bibliotecas y su importancia en la sociedad, para lograr el alcance de sus objetivos y los beneficios que otorga la existencia de las mismas en un contexto como el de los centros penitenciarios.

Por último, uno de los aspectos fundamentales del presente trabajo, consiste en atraer la atención sobre la función principal de los centros penitenciarios que, indudablemente es el de buscar un logro principal, de la manera más integralmente posible: regresar a la sociedad individuos rehabilitados y conscientes de las faltas cometidas, con el mayor ánimo de aportar de manera positiva al bien común de su grupo social $y$, definitivamente, la biblioteca tiene mucho que aportar a dicho fin. 


\section{Q ANEXO I}

\section{Formato de cuestionario a encargados de bibliotecas penitenciarias}

El presente cuestionario forma parte de un proyecto de investigación, cuyo objetivo es analizar la influencia de la figura de la biblioteca como instrumento para la reinserción social en los centros penitenciarios de Jalisco.

El presente instrumento está dirigido a los encargados de las bibliotecas que forman parte del Sistema Penitenciario del Estado de Jalisco.

Los resultados aquí obtenidos serán utilizados únicamente con fines científicos y se garantiza la confidencialidad de los datos, así como el anonimato de los participantes en el estudio.

Los resultados obtenidos en este instrumento serán compartidos con los participantes en el mismo, de manera que puedan aprovecharlos para fines académicos y de investigación.

Responder el presente cuestionario le tomará entre 8 y 10 minutos como máximo.

¡Muchas gracias por su valiosa colaboración!

Formación profesional:

Edad:

Género: Años en el puesto de encargado de biblioteca:

Nombre del centro penitenciario:

Por favor, responda a las siguientes preguntas:

1. ¿Qué es para usted una biblioteca?

2. ¿Qué servicios ofrece la biblioteca en la que usted labora:

1. Catálogo público impreso ()

2. Catálogo público en línea ()

3. Colección de libros ()

4. Hemeroteca ()

5. Computadora para el bibliotecario ()

6. Computadoras para los usuarios ()

7. Internet ()

8. Bases de datos académicas ()

9. Cursos de formación a usuarios ()

10. Sala de lectura ()

11. Mesas y sillas de trabajo para usuarios ()

12. Otros:

3. ¿Cuántas personas componen el personal de su biblioteca? 
4. ¿Cómo está compuesta la colección de su biblioteca?

5. ¿Cuál es el horario de servicios de su biblioteca?

6. ¿Considera que cuenta con los recursos necesarios para el funcionamiento de su biblioteca?

$$
\text { No _ Si _ ¿Por qué? }
$$

7. En promedio ¿Qué número de usuarios visita a diario su biblioteca?

Menos de 10 _ De 10 a 20_ De 20 a 30 _ Más de 30 _ Otro:

8. ¿Cuenta con un registro de sus usuarios?

No __ Si ___ ¿Cómo lo hace

9. ¿Existe el servicio de préstamo externo de materiales de la biblioteca?

No _ _ Si __ ¿Cómo lo hace?

10. ¿Ofrece cursos y/o actividades para los usuarios en su biblioteca?

No _

11. Si la respuesta anterior fue un sí ¿Qué cursos y/o actividades ofrece?

12. ¿Cuenta con algún programa de fomento a la lectura?

No _ _ Si __ ¿Cómo lo hace?

13. ¿Recibe algún tipo de capacitación para el desempeño de su puesto?

No _

14. ¿Ha recibido alguna formación para el trato con los reclusos?

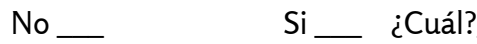

15. Para usted ¿Qué importancia juega la biblioteca en el proceso de reinserción social de los internos?

16. ¿Acuden a usted los usuarios de la biblioteca para resolver alguna duda?

No _

17. Si la respuesta anterior fue Sí ¿Cuáles son las consultas más frecuentes de sus usuarios? ¿Para qué lo buscan a usted?

18. Cuéntenos cómo ha sido su experiencia como encargado de una biblioteca penitenciaria:

19. ¿Algo más que quiera comentar?

¡Muchas gracias por su tiempo! 


\section{Referencias bibliográficas}

» Aristóteles. 1963 [2000]. Política. México: Universidad Nacional Autónoma de México.

"Cisneros, José Luis. 2016. Los factores psicosociales en el proceso de tratamiento para la readaptación social en las prisiones del Estado de México. En El Cotidiano. No. 200, 193-208. < https://www.redalyc.org/pdf/325/32548630017. pdf $>$ [Consulta: 2 Mayo 2020].

»Clemente Castro, Diana. 2019. El reconocimiento a la figura del bibliotecario a lo largo de la historia. Badajoz: Universidad de Extremadura. 79 p. Trabajo de grado. < http://dehesa.unex.es/handle/10662/9891> [Consulta: 2 Mayo 2020].

"Colás, María Pilar; Leonor Buendía y Fuensanta Hernández. 2009. Competencias científicas para la realización de una tesis doctoral. Guía metodológica de elaboración y presentación. Barcelona: Davinci Continental.

"Domínguez Sanjurjo, María Ramona. 1996. Nuevas formas de organización y servicios en la biblioteca pública. Gijón: Trea.

" Gill, Philip. 2002. Directrices IFLA/UNESCO para el desarrollo del servicio de bibliotecas públicas. México: Consejo Nacional para la Cultura y las Artes. <http:// eprints.rclis.org/7587/> [Consulta: 28 febrero 2020].

» Hernández Sampieri, Roberto; Carlos Fernández Collado y María del Pilar Baptista Lucio. 2014. Metodología de la investigación. México: McGraw Hill Interamericana.

»Jalisco. Gobierno del Estado. 2020. Fiscalía del Estado. <https://www.jalisco. gob.mx/gobierno/dependencias/fge> [Consulta: 28 febrero 2020]

"Lehmann, Vibeke y Joanne Locke. 2007. Pautas para servicios Bibliotecarios para reclusos. La Haya: IFLA Headquarters.

"Madrazo, Carlos. 1985. Educación, derecho y readaptación social. México: Instituto Nacional de Ciencias Penales.

" Marcos Recio, Juan Carlos. 2018. ¿Qué lugar ocupan los bibliotecarios en un mundo cada vez más colaborativo? En Morán Guzmán, Ana Griselda y Sergio López Ruelas, comp. Bibliotecarios: nuevas competencias, nuevas habilidades. México: Universidad de Guadalajara. p. 153-163.

» Meza, M. J. 1978. Readaptación social: Baja California Sur. La Paz: CERESO.

»México. Ley Nacional de Ejecución Penal. 2016. En Diario Oficial de la Federación, 16/o6/2016. <http://www.diputados.gob.mx/LeyesBiblio/pdf/LNEP_090518. pdf> [Consulta: 3 noviembre 2019].

" México. Constitución Política de los Estados Unidos Mexicanos. 2020. Última reforma, 06/03/2020. En Diario Oficial de la Federación, 05/02/1917. < https:// aafy.yucatan.gob.mx/legislaciones/Constitucion_Politica_de_los_Estados_ Unidos_Mexicanos.pdf> [Consulta: 26 enero 2020].

» Muñoz Blanco, Adriana G. 2004. La pena de muerte en México: Mitos y realidades. Guadalajara: Facultad de Derecho de la Universidad de Guadalajara. 147 p. Tesis de licenciatura. 
"Pérez Pulido, Margarita. 2007. Bibliotecas de prisiones: concepto, modelos y normas. En Educación y biblioteca. Vol. 19, no. 158, 73-81. <https://gredos.usal. es/handle/10366/119332> [Consulta: 20 noviembre 2019].

»Rousseau, Jean Jacques. 2014. Contrato social. Madrid: Biblioteca Nueva.

"Ruibal, María Isabel. 2018. Hablamos de...las bibliotecas de los centros penitenciarios. En Desiderata. No. 8, 34-41. <https://dialnet.unirioja.es/servlet/ articulo?codigo $=6366145>$ [Consulta: 10 diciembre 2019].

»UNESCO; IFLA Federación Internacional de Asociaciones de Bibliotecarios y Bibliotecas. 1994. Manifiesto de la UNESCO sobre la biblioteca pública. <http:// www.bpej.udg.mx/sites/default/files/manifesto_Biblioteca_publica_unesco. pdf> [Consulta: 8 enero 2020]. 